Supporting Information on:

\title{
Detection of Chemical Warfare Agents by Colorimetric Sensor Arrays
}

Charles E. Davidson, ${ }^{\dagger}$ Melissa M. Dixon, ${ }^{\ddagger}$ Barry R. Williams, ${ }^{\S}$ Gary K. Kilper, ${ }^{\perp}$ Sung H. Lim,॥

Raymond A. Martino,\| Paul Rhodes, $\|$ Melissa S. Hulet, ${ }^{\S}$ Ronald W. Miles, ${ }^{\ddagger}$ Alan C. Samuels, ${ }^{\ddagger}$ Peter

A. Emanuel, ${ }^{\ddagger}$ and Aleksandr E. Miklos ${ }^{\ddagger *}$

†Science and Technology Corporation, 111 C Bata Boulevard, Belcamp, MD 21017.

‡U.S. Army CCDC Chemical Biological Center, Aberdeen Proving Ground, MD 21010.

\$Leidos, 3465 Box Hill Corporate Center Drive, Abingdon, MD 21009.

${ }^{\perp}$ Excet, 6225 Brandon Avenue, Suite 360, Springfield, VA 22150.

\|iSense, 855 Maude Avenue, Mountain View, CA 94043.

Table S1. Agent Compounds

\begin{tabular}{|c|c|c|c|c|c|c|c|}
\hline \multirow{2}{*}{$\begin{array}{l}\text { Agent } \\
\text { Type }\end{array}$} & \multicolumn{2}{|c|}{ Abbreviation } & \multirow[b]{2}{*}{ Agent Name } & \multicolumn{4}{|c|}{ CSAs Exposed } \\
\hline & Type & Agent & & Train & Test & Total & $\begin{array}{l}\text { Type } \\
\text { Total }\end{array}$ \\
\hline \multirow{4}{*}{ Nerve/G } & G & GA & Tabun, (RS)-ethyl N,N-dimethyl-phosphoramidocyanidate & 5 & 10 & 15 & \multirow{4}{*}{110} \\
\hline & G & GB & Sarin, (RS)-propan-2-yl methyl phosphonofluoridate & 15 & 30 & 45 & \\
\hline & G & GD & Soman, 3,3-dimethylbutan-2-yl methylphosphonofluoridate & 10 & 15 & 25 & \\
\hline & G & GF & Cyclosarin, cyclohexyl methylphosphonofluoridate & 15 & 10 & 25 & \\
\hline \multirow{2}{*}{ Nerve/V } & $\mathrm{V}$ & VR & $\begin{array}{l}N, N \text {-Diethyl-2-(methyl-(2-methylpropoxy)phosphoryl) } \\
\text { sulfanylethanamine }\end{array}$ & 3 & 5 & 8 & \multirow[b]{2}{*}{38} \\
\hline & V & vX & $\begin{array}{l}\text { Ethyl(\{2-[bis(propan-2-yl)amino]ethyl }\} \text { sulfanyl)(methyl) } \\
\text { phosphinate }\end{array}$ & 5 & 25 & 30 & \\
\hline \multirow{5}{*}{ Mustard } & $\mathrm{H}$ & HD & Bis(2-chloroethyl)ethylamine & 10 & 15 & 25 & \multirow{5}{*}{75} \\
\hline & $\mathrm{H}$ & $\mathrm{HN}_{1}$ & Nitrogen Mustard 1, bis(2-chloroethyl)ethylamine & 5 & 10 & 15 & \\
\hline & $\mathrm{H}$ & $\mathrm{HN}_{3}$ & Nitrogen Mustard 3, tris (2-chloroethyl)amine & 5 & 5 & 10 & \\
\hline & $\mathrm{H}$ & HT & Bis(chloroethyl)ether & 5 & 15 & 20 & \\
\hline & $\mathrm{H}$ & $\mathrm{T}$ & Bis(2-chloroethylthioethyl)ether & 5 & o & 5 & \\
\hline \multirow{3}{*}{ Arsenical } & $\mathrm{L}$ & L-lewisite & Lewisite, 2-chlorovinyldichloroarsine & 5 & 5 & 10 & \multirow{3}{*}{25} \\
\hline & $\mathrm{L}$ & $\mathrm{L} 2$ & Bis(2-chloroethenyl)arsinous chloride & 5 & 5 & 10 & \\
\hline & $\mathrm{L}$ & $\mathrm{L}_{3}$ & Tris(2-chlorovinyl)arsine & 5 & o & 5 & \\
\hline \multirow{7}{*}{$\begin{array}{l}\text { Precursor/ } \\
\text { byproduct }\end{array}$} & $\mathrm{P} / \mathrm{B}$ & CEES & 2-Chloroethyl ethyl sulfide & 5 & o & 5 & \multirow{7}{*}{56} \\
\hline & $\mathrm{P} / \mathrm{B}$ & DIAET & 2-(Diisopropylamino)ethanethiol & 5 & 10 & 15 & \\
\hline & $\mathrm{P} / \mathrm{B}$ & DiCDi & 1,3-Diisopropylcarbodiimide & 5 & 5 & 10 & \\
\hline & $\mathrm{P} / \mathrm{B}$ & DIMP & Diisopropyl methylphosphonate & 4 & 5 & 9 & \\
\hline & $\mathrm{P} / \mathrm{B}$ & DMMP & Dimethyl methylphosphonate & 3 & 4 & 7 & \\
\hline & $\mathrm{P} / \mathrm{B}$ & EMPA & Ethyl methylphosphonic acid & 5 & o & 5 & \\
\hline & $\mathrm{P} / \mathrm{B}$ & QL & Isopropyl aminoethylmethyl phosphonite & 5 & o & 5 & \\
\hline
\end{tabular}


Table S2. Non-Agent Compounds

\begin{tabular}{|c|c|c|c|c|c|c|c|}
\hline \multirow{2}{*}{$\begin{array}{c}\text { Chemical } \\
\text { Type }\end{array}$} & \multicolumn{2}{|c|}{ Abbreviation } & \multirow[b]{2}{*}{ Chemical Name } & \multicolumn{4}{|c|}{ CSAs Exposed } \\
\hline & Type & Chemical & & Train & Test & Total & $\begin{array}{c}\text { Type } \\
\text { Total } \\
\end{array}$ \\
\hline \multirow{12}{*}{$\begin{array}{l}\text { General } \\
\text { Chemical }\end{array}$} & 1 & Cyanamide & Cyanamide (50\% wt. solution in water) & 5 & o & 5 & \multirow{12}{*}{75} \\
\hline & 1 & DCP & Diethylchlorophosphate & 3 & 3 & 6 & \\
\hline & 1 & DEA & Diethylamine & 3 & 5 & 8 & \\
\hline & 1 & DEG & Diethylguanidine (sulfate salt) & 3 & o & 3 & \\
\hline & 1 & $\mathrm{EtOH}$ & Ethanol & 3 & 3 & 6 & \\
\hline & 1 & $\mathrm{HCl}$ & Hydrochloric acid & 3 & 8 & 11 & \\
\hline & 1 & IPA & Isopropanol & 3 & 3 & 6 & \\
\hline & 1 & $\mathrm{KOH}$ & Potassium hydroxide & 3 & 3 & 6 & \\
\hline & 1 & $\mathrm{MeCl}$ & Dichloromethane & 3 & 3 & 6 & \\
\hline & 1 & $\mathrm{MeS}$ & Methyl salicylate & 3 & o & 3 & \\
\hline & 1 & TEA & Triethylamine & 3 & 3 & 6 & \\
\hline & 1 & TEOA & Triethanolamine & 4 & 5 & 9 & \\
\hline \multirow{3}{*}{ Insecticide } & 2 & Fipronil & Fipronil (Termidor ${ }^{\mathrm{TM}}$ liquid concentrate) & 5 & 5 & 10 & \multirow{3}{*}{30} \\
\hline & 2 & Malathion & Malathion & 5 & 5 & 10 & \\
\hline & 2 & Permethrin & Permethrin & 5 & 5 & 10 & \\
\hline \multirow{3}{*}{ Herbicide } & 3 & Roundup & Roundup $^{\mathrm{TM}}$ glyphosate-based, liquid concentrate & 5 & 9 & 14 & \multirow{3}{*}{39} \\
\hline & 3 & WBG-orange & Ortho Weed-B-Gon ${ }^{\mathrm{TM}}$ crabgrass control concentrate & 5 & 5 & 10 & \\
\hline & 3 & WBG-purple & Ortho Weed B Gon ${ }^{\mathrm{TM}}$ chickweed, clover and oxalis killer & 10 & 5 & 15 & \\
\hline \multirow{8}{*}{ Household } & 4 & Bleach & Bleach (hypochlorite) & 4 & 5 & 9 & \multirow{8}{*}{70} \\
\hline & 4 & DirtDevil & Dirt Devil $^{\mathrm{TM}}$ carpet shampoo & 4 & 5 & 9 & \\
\hline & 4 & GoofOff & Goof Off ${ }^{\mathrm{TM}}$ adhesive remover solvent & 5 & 4 & 9 & \\
\hline & 4 & Hoppes 9 & Hoppes No.9 ${ }^{\mathrm{TM}}$ small arms cleaning solvent & 5 & 5 & 10 & \\
\hline & 4 & Lysol & Lemon-scented Lysol ${ }^{\mathrm{TM}}$ cleaning product & 4 & o & 4 & \\
\hline & 4 & Pine-Sol & Pine-Sol ${ }^{\mathrm{TM}}$ cleaning product & 5 & 5 & 10 & \\
\hline & 4 & Simple Green & Simple Green ${ }^{\mathrm{TM}}$ cleaning product & 4 & 5 & 9 & \\
\hline & 4 & Windex & Windex ${ }^{\mathrm{TM}}$ cleaning product & 5 & 5 & 10 & \\
\hline \multirow{9}{*}{ Miscellaneous } & 5 & ATF & Automatic transmission fluid (automotive) & 5 & 10 & 15 & \multirow{9}{*}{114} \\
\hline & 5 & CLP & $\mathrm{CLP}^{\mathrm{TM}}$ small arms cleaner/lubricant & 2 & o & 2 & \\
\hline & 5 & WD-40 & WD- $40^{\mathrm{TM}}$ general-purpose lubricant & 5 & o & 5 & \\
\hline & 5 & JP8 & Jet propellant 8(kerosene based) & 4 & 5 & 9 & \\
\hline & 5 & AFFF & Aqueous film forming foam (fire-fighting foam) & 5 & 5 & 10 & \\
\hline & 5 & Antifreeze & Propylene glycol automotive antifreeze & 4 & 5 & 9 & \\
\hline & 5 & Bengay & Bengay Ultra ${ }^{\mathrm{TM}}$ topical analgesic & 5 & o & 5 & \\
\hline & 5 & Grass & Cut grass (turf grass, cut with scissors, flash-frozen) & 4 & 5 & 9 & \\
\hline & 5 & Blank & Unexposed & 24 & 26 & 50 & \\
\hline
\end{tabular}

\section{S-1. EXTRACTING RGB DATA FROM CSA IMAGES}

In this section, a description of the image processing steps to extract RGB data from CSA imagery is presented. The first processing step was to locate and align the ticket, which eliminated uncertainty in ticket location, orientation, and size in the image. Each ticket had three chemically inactive control spots that were referenced to determine the CSA scale and orientation in the image. Morphological processing and threshold segmentation were used to identify the control spots. For the scanner, the imaging axis was normal to the CSA surface; therefore, we assumed no perspective distortion, and a simple geometric analysis of the control spot centroids determined a rotation angle for aligning the image and a rectangular region for tightly cropping to the CSA borders (see Figure 2, b and c).

The second step was spot detection, whereby the aligned image was broken into a grid of tiles, such that each spot was contained within a single tile. A statistical anomaly detection algorithm (Mahalanobis distance) was applied to each tile to identify pixels dissimilar from the surrounding (local) background. Spatial processing and morphological reconstruction operators were used to 
refine the spot boundaries. For a central tile in the grid, Figure $2 \mathrm{~d}$ highlights the local region from which background statistics were estimated. Figure $2 \mathrm{e}$ shows the value of the anomaly detection statistic for each pixel in the image, and Figure $2 \mathrm{f}$ shows identified spot boundaries.

Finally, the extraction step eroded an outer margin (so that only the central portion of each spot was considered) and computed RGB color values for each spot. Statistics such as the mean RGB vector $\left(\boldsymbol{\mu}_{1}\right)$ and the mean RGB vector of surrounding background areas $\left(\boldsymbol{\mu}_{\mathrm{o}}\right)$ were computed for each spot. The result of the processing was a time series of statistics for each spot across all images of the CSA. For the $i^{\text {th }}$ trial, consisting of RGB source images (where the number of channels, $c$, was 3 ) observing a CSA with $N$ spots, over $T_{i}$ time points, $\boldsymbol{\mu}_{\mathrm{o}}$ and $\boldsymbol{\mu}_{1}$ may each be naturally organized as a ${ }_{3} \mathrm{D}$ numeric array of size $c$ by $N$ by $T_{i}$. It was also convenient to combine the spot and channel dimensions into a single vector that characterizes the color response of the entire CSA at a given time point, i.e., by reorganizing the $\boldsymbol{\mu}_{1}$ and $\boldsymbol{\mu}_{\mathrm{o}}$ arrays into $(c \times N)$ by $T_{i}$ arrays (i.e., stacking the RGB triples for all spots at a given time). We referred to each element of the stacked vector as a "band". For the scanner images of the sensors, $c=3$ and $N=73$, which resulted in a 219 by $T_{i}$ matrix of color values for the ith test fixture. The last row in Figure 2 is an image representation of the $\boldsymbol{\mu}_{1}$ values at a single time point (Figure $2 \mathrm{~g}$ ) and the stacked color-vector representation (Figure $2 \mathrm{~h}$ ).

Extracted RGB averages may be analyzed as is; however, certain additional processing steps may be warranted:

1. Basic illumination correction: White-balancing each spot with respect to the white areas of the ticket (the background) forms a relative reflectance array, $\boldsymbol{\rho}=\boldsymbol{\mu}_{1} / \boldsymbol{\mu}_{\mathrm{o}}$. White-balancing converts to relative reflectance and normalizes for illumination changes such as source intensity. Local white-balancing can help correct for changes in the physical conformation of the CSA: the backing material may occasionally swell or pucker, which changes the spatial relationship between the surface normal and the light source across the ticket and causes local appearance changes that are compensated for by the procedure.

2. Statistical "whitening" (not to be confused with white-balancing): The covariance matrix of the relative reflectance array for all pre-exposed images of all training tickets, $\boldsymbol{\Sigma}=\operatorname{cov}[\boldsymbol{\rho}(\mathrm{t} \leq \mathrm{o})]$, is a 219 by 219 matrix that measures the spread caused by variability in the CSAs and the digital imageprocessing pathway. Whitening the data by multiplying each 219-dimensional vector by the matrix square root of the covariance matrix, $\mathbf{y}_{i}=\Sigma^{-1 / 2} \boldsymbol{\rho}_{i}$, transforms the original measurement variables (bands) to form a new set of coordinate axes that are proportional to the signal-to-noise ratio and are therefore more statistically meaningful. Euclidean distances in the transformed space are equivalent to Mahalanobis distances in the original space. Note that the term "white" in multivariate statistics means that the error distribution is isotropic- error magnitude is the same in all directions - and was coined in analogy to white light, which is composed of all colors. It does not describe nor should it be confused with the appearance of the CSAs. We are careful to use the phrase "statistical whitening" to avoid confusion.

3. Computing the difference to the pre-exposure response: $\Delta(\mathrm{t})=\boldsymbol{\rho}(\mathrm{t})-\boldsymbol{\rho}(\mathrm{t}=\mathrm{o})$, or if whitening is applied, $\Delta(\mathrm{t})=\mathrm{y}(\mathrm{t})-\mathrm{y}(\mathrm{t}=\mathrm{o})$. This is a measure of the color change for each spot, and it can help correct for non-uniformities from ticket to ticket. For example, two tickets might exhibit different RGB averages for a spot if a dust mote were to occlude a portion of the spot on ticket 1 but not on ticket 2. However, the color-change direction will be unaffected by the presence of the (stationary) dust mote: the RGB average at time $t=0$ and at a later time $t$ will both be affected equally. Time $t=\mathrm{o}$ subtraction may be problematic, however, for any spot that shows a large variance in appearance under an unexposed state. For example, assume that a particular pigment appears sometimes red and sometimes yellow when the ticket is unexposed. If exposure to a particular chemical results in that spot becoming red, in one case, there will be no color change associated 
with the exposure, but in the other case, there will be an observed color change. Without subtracting the time $t=0$ response, the ending result is consistent: exposure to the chemical results in a red spot. Therefore, there is a trade-off in forming the color-change result that will be helpful in some cases but harmful in others.

4. Interpolation to a common elapsed time axis: Test fixtures were collected at varying sampling intervals ( 2 or $5 \mathrm{~min}$ ) and durations (usually $1-2$ hours). To enable concatenation of all data into a single multidimensional array, each test fixture was linearly interpolated to a 2 min interval. In this paper, we limit the time period of interest from 2 to $54 \mathrm{~min}$.

Unless otherwise noted, all preprocessing steps were applied to the data presented herein.
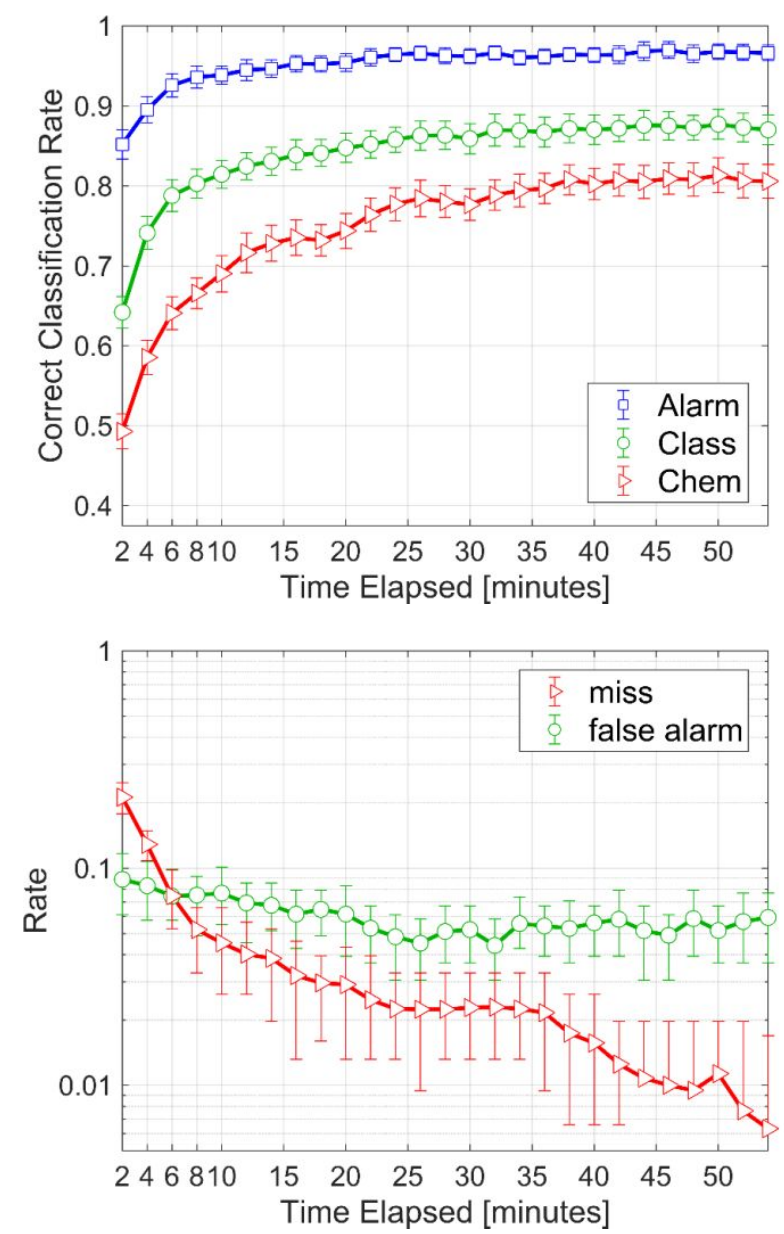

Figure S1. Classification performance over time as estimated using cross-validation. Top: average classification rate at 3 levels of stringency. Error bars show +/- 1 standard deviation. Bottom: average false alarm (declaring a non-threat chemical a chemical weapon) and missed detection rates (declaring a chemical weapon as a non-threat). 


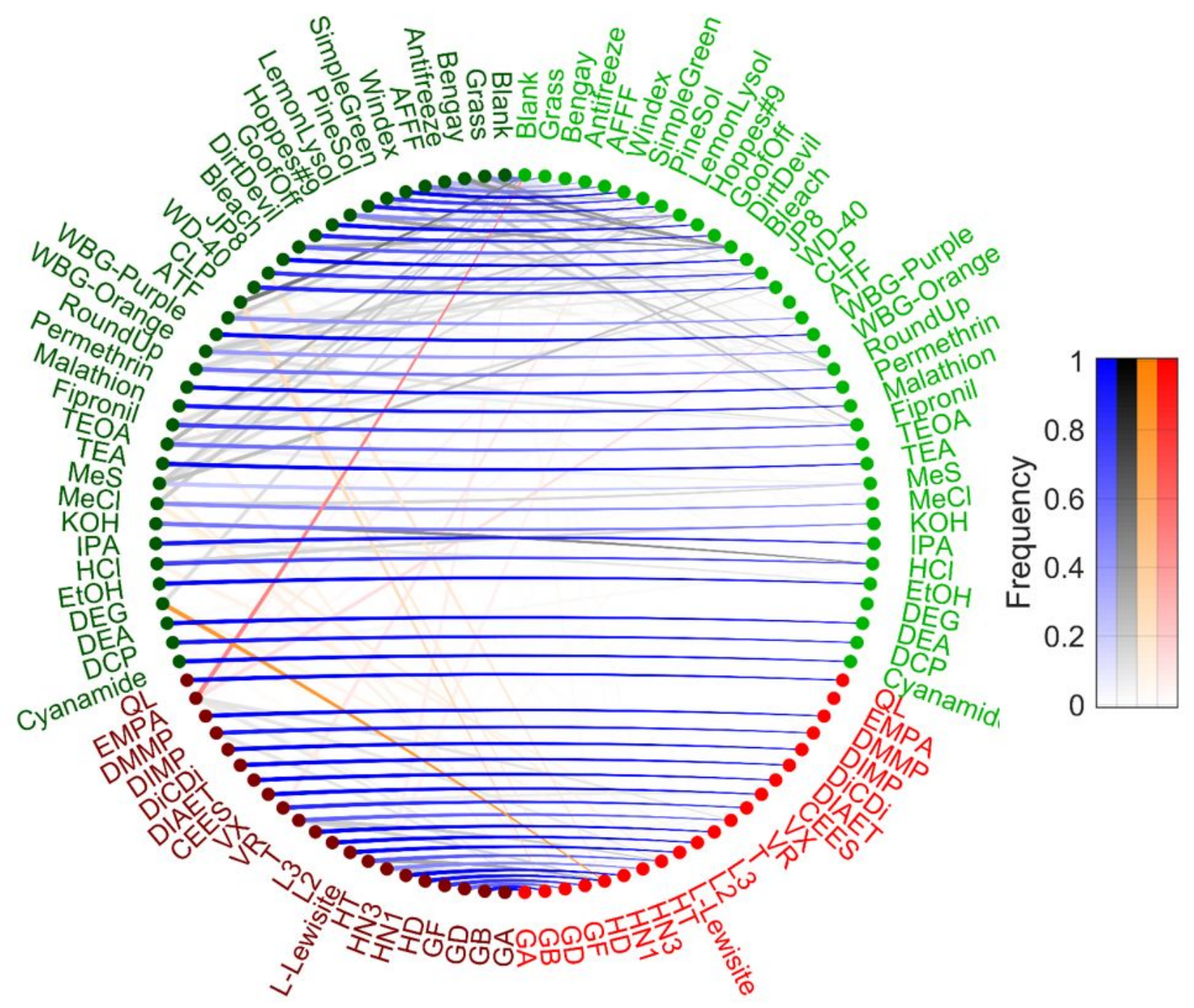

Figure S2. Graphical representation of confusion rates at chemical-level identification for 30 minutes elapsed as estimated by the cross validation procedure. Nodes around the left half of the circle represent the true exposure. Directed edges point to the node(s) along the right half of the circle giving the declared or predicted chemical. There are potentially four edges emanating from each node: blue edges point to the correct chemical; black edges point to the wrong chemical but correct chemical class; orange edges point from a non-agent chemical to an agent; red edges point from an agent to a non-agent chemical. The color intensity is determined by the frequency of occurrence. The most obvious misidentifications include $80 \%$ of DEG exposures misidentified as HD (a false alarm) and 48\% of EMPA exposures identified as blank (a missed detection). 


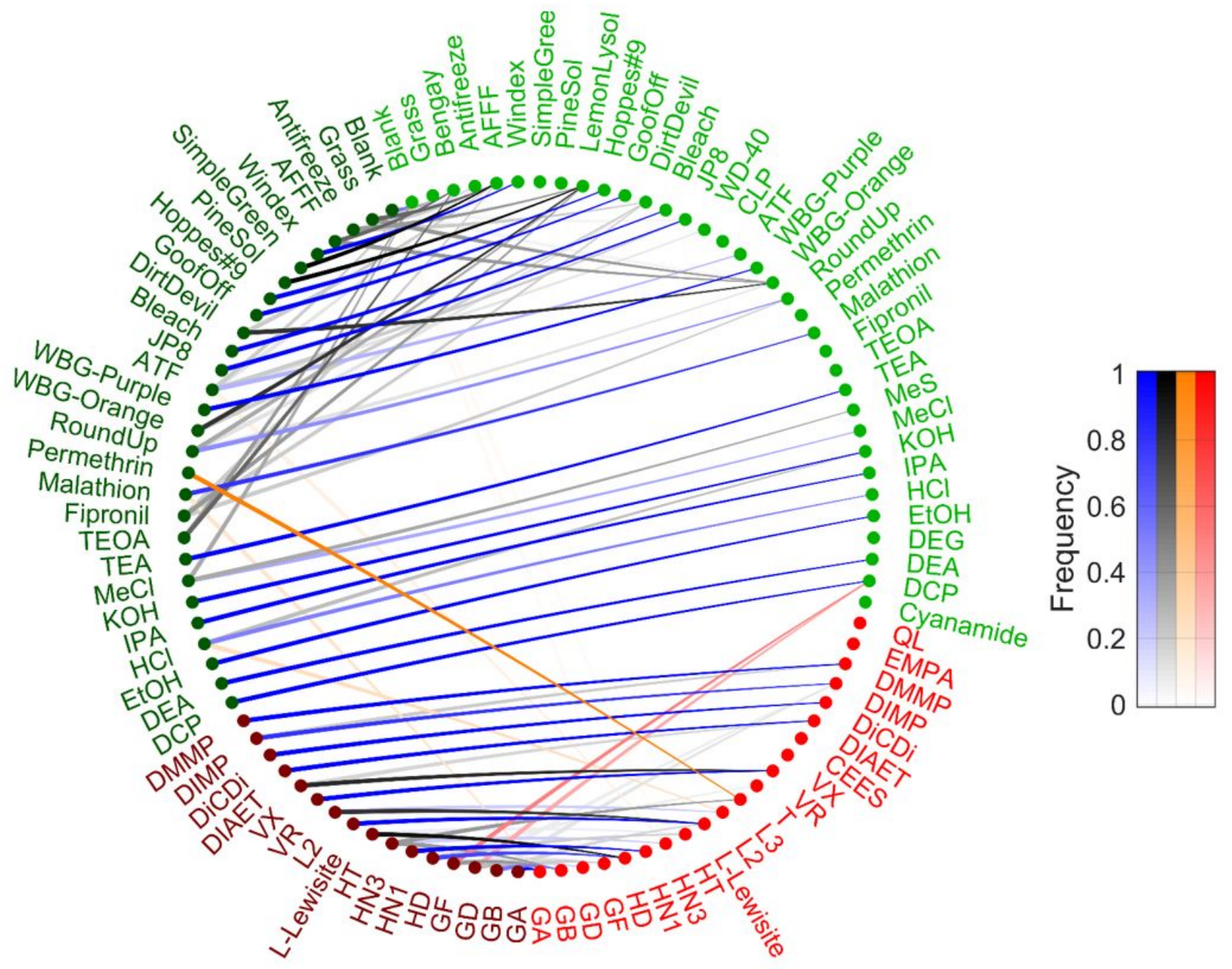

Figure S3. Graphical representation of confusion rates at chemical-level identification for 30 minutes elapsed as estimated by the independent test set. Nodes around the left half of the circle represent the true exposure. Note that exposures without representation in the test set do not have nodes along the left side of the graph. Directed edges point to the node(s) along the right half of the circle giving the declared or predicted chemical. There are potentially four edges emanating from each node: blue edges point to the correct chemical; black edges point to the wrong chemical but correct chemical class; orange edges point from a non-agent chemical to an agent; red edges point from an agent to a non-agent chemical. The color intensity is determined by the frequency of occurrence. 
Table S3. Average Class Confusion Matrix at 30 Min Elapsed Time as Estimated by Cross-Validation

\begin{tabular}{r|ccccc|ccccc}
\multicolumn{1}{c}{} & $\mathrm{G}$ & $\mathrm{V}$ & $\mathrm{H}$ & $\mathrm{L}$ & $\mathrm{P} / \mathrm{B}$ & 1 & 2 & 3 & 4 & 5 \\
\cline { 2 - 10 } $\mathrm{G}$ & $\mathbf{0 . 9 7 4}$ & 0.018 & & 0.002 & 0.003 & & 0.003 & 0.0004 & & \\
$\mathrm{~V}$ & & $\mathbf{0 . 9 7 5}$ & & & & & & 0.001 & 0.024 & \\
$\mathrm{H}$ & 0.041 & & $\mathbf{0 . 9 3 7}$ & 0.004 & 0.001 & & & 0.003 & 0.006 & 0.009 \\
$\mathrm{~L}$ & & & & $\mathbf{0 . 9 8 8}$ & & 0.008 & 0.003 & & & \\
$\mathrm{P} / \mathrm{B}$ & 0.026 & 0.006 & 0.023 & 0.003 & $\mathbf{0 . 8 8 6}$ & & & & 0.001 & 0.055 \\
\hline 1 & 0.005 & & 0.054 & 0.021 & 0.002 & $\mathbf{0 . 8 1 3}$ & & & 0.007 & 0.100 \\
2 & 0.031 & & & 0.007 & & & $\mathbf{0 . 8 7 7}$ & 0.036 & 0.035 & 0.015 \\
3 & & 0.001 & & & & & 0.055 & $\mathbf{0 . 7 2 3}$ & 0.188 & 0.033 \\
4 & 0.001 & & 0.003 & & 0.003 & 0.010 & 0.019 & 0.037 & $\mathbf{0 . 8 8 4}$ & 0.043 \\
5 & 0.015 & 0.0004 & 0.070 & & 0.005 & 0.028 & 0.038 & 0.026 & 0.145 & $\mathbf{0 . 6 7 3}$
\end{tabular}

Element $(i, j)$ gives the fraction of exposures with true class $i$ were predicted to be class $j$. The upper-right quadrant of the table gives class-conditional agent miss rates. The lower-left quadrant gives false-alarm rates. Misidentification rates of $o$ are suppressed and represented by an empty cell.

Table S4. Class Confusion Matrix at 30 Min Elapsed Time for Independent Test Set

\begin{tabular}{r|ccccc|ccccc}
\multicolumn{1}{l}{} & $\mathrm{G}$ & $\mathrm{V}$ & $\mathrm{H}$ & $\mathrm{L}$ & $\mathrm{P} / \mathrm{B}$ & $\mathbf{1}$ & 2 & 3 & 4 & 5 \\
\cline { 2 - 10 } $\mathrm{G}$ & $\mathbf{0 . 7 0 8}$ & & & 0.077 & 0.062 & 0.154 & & & & \\
$\mathrm{~V}$ & & $\mathbf{0 . 8 6 7}$ & & & 0.133 & & & & & \\
$\mathrm{H}$ & 0.044 & & $\mathbf{0 . 9 1 1}$ & 0.044 & & & & & & \\
$\mathrm{~L}$ & & & & $\mathbf{1 . 0 0 0}$ & & & & & & \\
$\mathrm{P} / \mathrm{B}$ & & & & & $\mathbf{1 . 0 0 0}$ & & & & & \\
\hline 1 & & & & 0.056 & & $\mathbf{0 . 7 7 8}$ & & & & 0.167 \\
2 & 0.067 & & & 0.333 & & & $\mathbf{0 . 2 6 7}$ & 0.067 & 0.200 & 0.067 \\
3 & & & & & & & & $\mathbf{0 . 5 2 6}$ & 0.474 & \\
4 & & & & & & & & 0.118 & $\mathbf{0 . 7 0 6}$ & 0.176 \\
5 & & & 0.054 & & & & & 0.089 & 0.054 & $\mathbf{0 . 8 0 4}$
\end{tabular}




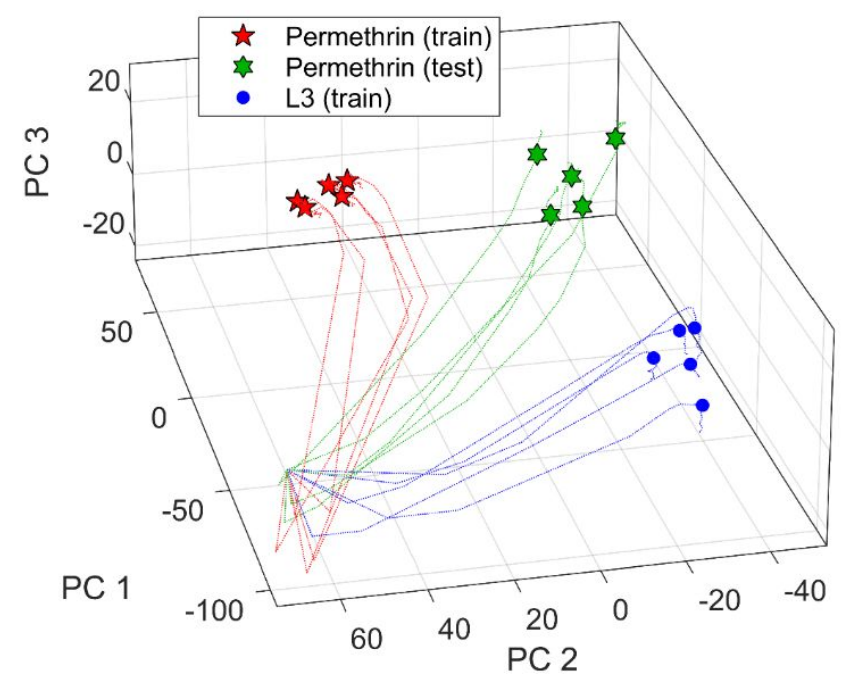

Figure $\mathrm{S}_{4}$. Visualization of color change in a $3 \mathrm{D}$ principal component space for permethrin and $\mathrm{L}_{3}$ exposures. Curves represent the color change track up to 54 minutes elapsed; markers specify the location of the color change at 30 minutes. All curves emanate from a point in the lower left corner of the figure, which represents no change. The permethrin testing exposures (from a survey experiment) were all more similar to one another than to either the permethrin or the L3 training exposures. The color changes identified on several spots of the testing exposures were intermediate between those for the L 3 and permethrin training exposures. The differences were such that L3 was slightly closer than permethrin (in terms of Euclidean distance), and the testing trials were misidentified (see Figure 9). Both experiments were exposed using the same permethrin source, but they were executed a year apart and involved different CSA batches (from the manufacturer). 


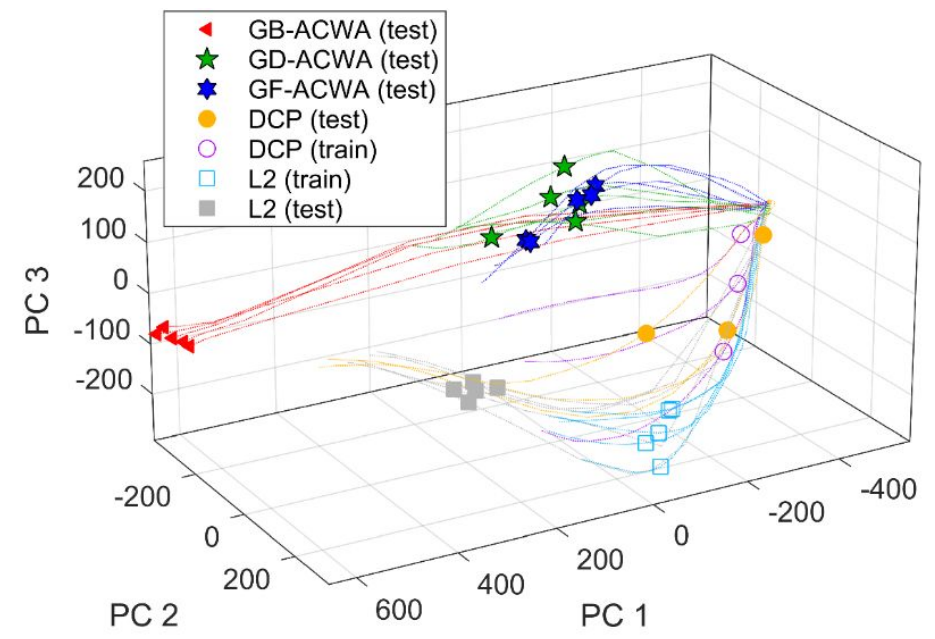

Figure $\mathrm{S}_{5}$. Visualization of color change in a $3 \mathrm{D}$ principal component space for cluster 10 exposures (see Figure 4 and Table 3). Curves represent color-change track up to 54 min elapsed; markers specify locations of color changes at $30 \mathrm{~min}$. All curves emanate from a point to the right of the figure which represents no change. All G agent samples from this cluster resembled one another, although GB changed more quickly and intensely. However, all of these G exposures were part of the test set. The closest neighbor from the training set for GD and GF exposures at 30 min was DCP, whereas GB was in fact closer to L-lewisite (a member of cluster 11 and not shown in this plot). These $\mathrm{G}$ agents were all obtained from the same source and are suspected to be lower purity. 